\title{
TITLE:
}

\section{Hydration Structure in Dilute Hydrofluoric Acid}

$\operatorname{AUTHOR}(S)$ :

Joutsuka, Tatsuya; Ando, Koji

CITATION:

Joutsuka, Tatsuya ... [et al]. Hydration Structure in Dilute Hydrofluoric Acid. The Journal of Physical Chemistry A 2011, 115(5): 671-677

ISSUE DATE:

2011-01-06

URL:

http://hdl.handle.net/2433/217072

\section{RIGHT:}

This document is the Accepted Manuscript version of a Published Work that appeared in final form in The 'Journal of Physical Chemistry A', copyright ( $)$ American Chemical Society after peer review and technical editing by the publisher. To access the final edited and published work see http://dx.doi.org/10.1021/jp108147e.; この論文は出版社版でありま せん。引用の際には出版社版をご確認ご利用ください。; This is not the published version. Please cite only the published version. 


\title{
Hydration Structure in Dilute Hydrofluoric Acid
}

\author{
Tatsuya Joutsuka and Koji Ando \\ Department of Chemistry, Kyoto University, Sakyo-ku, Kyoto 606-8502, Japan
}

\begin{abstract}
We have performed the multistate empirical valence bond (MS-EVB) molecular dynamics simulations of a dilute hydrofluoric acid solution at ambient temperature to study the hydration structure associated with its weak acidity. The developed MS-EVB model showed reasonable agreement with experiment and previous ab initio molecular dynamics and reference interaction site model self-consistent field simulations for the free energy and structural properties. The local tetrahedral and translational order parameters around the fluorine atom significantly increase in the transition and product states of the HF dissociation reaction. This indicates that the angular and translational rearrangements of the hydrogen-bond topology are necessary especially around the fluorine atom. At the transition state of the proton transfer, the tetrahedral order parameters are very large, whereas the translational order parameters are not. This suggests that for the proton transfer to occur the large angular rearrangements of the hydrogen-bond topology are more necessary than the translational ones.
\end{abstract}

\section{Introduction}

A molecular-level description of aqueous acid ionization is pivotal for elementary acid-base chemistry in water. ${ }^{1-4}$ Particularly, the series of hydrohalic acids ( $\mathrm{HX}$, where $\mathrm{X}=\mathrm{F}, \mathrm{Cl}, \mathrm{Br}$, and I), with the acidity increasing toward the heavier halides, presents a representative model for the study of acidity in water. ${ }^{5}$ Among these acids, the hydrofluoric acid has peculiar properties; for example, at low concentrations aqueous HF is a weak acid whose acidity and solubility continues to increase along the concentration whereas those of ordinary weak acids reach a constant value. ${ }^{6}$ The reasons for these behaviors have fuelled controversies over the years. ${ }^{7-11}$

Notably, the counterintuitive fact that the weak acid dissociates more at a lower temperature $^{12}$ reflects that the large negative entropic contribution to the dissociation free energy is one of the main factors in determining the weak acidity of a dilute hydrofluoric acid solution. ${ }^{9,13}$ This contribution is much more pronounced than that for the other members of the series ${ }^{10}$ because the electrostatic attractiveness of the small fluoride ions imposes a substantial order on the surrounding water molecules as well as nearby hydroxonium ions, whose effect falls for larger halide ions. This behavior might be ascribed to the "structure making" character of $\mathrm{F}^{-}$hydration in water, whereas the bigger "structure breaker" halide anions $\mathrm{Cl}^{-}, \mathrm{Br}^{-}$, and $\mathrm{I}^{-}$disrupt the hydrogen-bond (H-bond) network of liquid water. ${ }^{14}$

In contrast with the above speculation, a recent series of pump-probe and terahertz spectroscopies and molecular dynamics (MD) simulations have suggested that halide anions may not be considered as the "kosmotropes (structure makers)" nor "chaotropes (structure breakers)" "15-17 that affect the dynamics of water molecules outside the first solvation shells of the 
ions. Instead, they could be regarded as "rigid spheres" formed by the ion and its first solvation shell in a picosecond time scale. On the other hand, this picture has been challenged by MD simulation by Laage et al., who have found a labile hydration sphere. ${ }^{18}$ These recent findings suggest that the change of the hydration structure, and consequently the anomalous entropy change, can be related to the solvent reorganization near the HF molecule, in particular to its first and second hydration shells.

The purpose of this paper is to analyze the hydration structure during the proton transfer (PT) reaction associated with the weak acidity in a dilute hydrofluoric acid solution. For this purpose, equilibrium MD simulation is performed by developing the multistate empirical valence bond (MS-EVB) model. ${ }^{19,20}$ The nuclear quantum effect (NQE) is approximately included by fitting the effective potential of mean force from the centroid molecular dynamics (CMD) approach, ${ }^{21}$ in which the potential energy surface (PES) is quantized at a given temperature by a preliminary path-integral calculation. ${ }^{22-24}$ We present the parametrization of our model for the $\mathrm{HF}-\mathrm{H}_{2} \mathrm{O}$ dimer and apply it to the equilibrium MD simulations. To investigate the hydration structure in detail, we report the H-bond topology by calculating the local tetrahedral and translational order parameters, which are often used to quantify structural order for the anomalies of liquid water. ${ }^{25,26}$

This paper is organized as follows. In section 2, the details of molecular model employed in this study are summarized. In section 3, the results are presented and discussed. Specifically, in sections 3.1 and 3.2, the free energies and radial distribution functions (RDFs) of the reaction are discussed. In section 3.3, we analyze the H-bond configuration change associated with the weakness of HF acid in the reaction in terms of the order parameters. Section 4 concludes.

\section{Molecular Model Details}

\subsection{Multistate Empirical Valence Bond Model}

The multistate empirical valence bond model (MS-EVB) has been described previously in detail. ${ }^{27-32}$ In the following, we briefly review the general idea and then describe the characteristic aspects in the present study. The MS-EVB model allows a proton sharing between HF and water molecules and the Hamiltonian is given by

$$
H_{\mathrm{EVB}}=\sum_{i, j}|i\rangle h_{i j}\langle j|
$$

where $h_{i i}$ describes the potential energy of the valence bond (VB) state $|i\rangle$ and $h_{i j}$ is the coupling between the VB states $|i\rangle$ and $|j\rangle$. Figure 1 illustrates the implementation of MS-EVB for $N$ VB states. The criteria in the simulation for selecting an EVB state are that the $\mathrm{H}_{\mathrm{HF}}-\mathrm{O}_{\mathrm{w}}$ (w means water) distance is less than $6.1 \AA$ and the $\mathrm{F}_{\mathrm{HF}}-\mathrm{H}_{\mathrm{HF}}-\mathrm{O}_{\mathrm{w}}$ angle is greater than $60^{\circ}$. This procedure ensures that the proton can be transferred only to water molecules included in the EVB complex. This approximation can be justified by the suggestion by IR experiments and Car-Parrinello molecular dynamics (CPMD) simulations ${ }^{34-38}$ that the proton is rarely transferred further than the nearest-neighbor water molecule. A total of 5-9 EVB states are typically included during the simulation indicating that the multistate description of VB states are essential. ${ }^{31,32}$ To determine the energy, the lowest eigenvalue for the Hamiltonian matrix is evaluated, and the HellmanFeynman forces are used. 


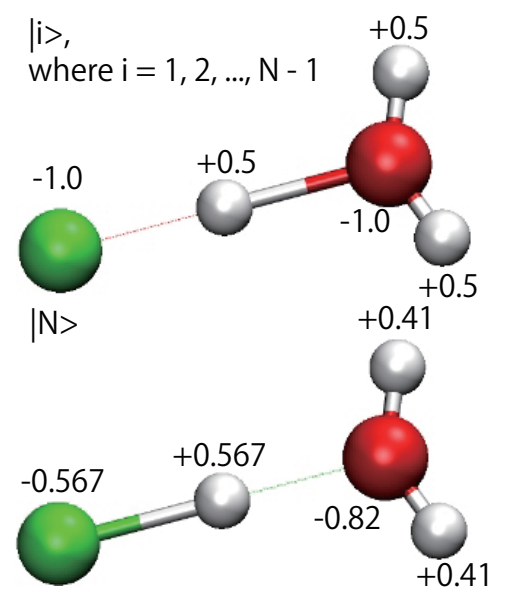

Figure 1: Illustration of the formation of possible $N$ valence bond (VB) states. The upper panel shows the contact ion pair VB state, whereas the lower one displays the covalent VB state. The value near each atom is the point charge for the respective atom. The dotted lines represent the $\mathrm{H}$-bonds. The graphic was drawn with the VMD program. ${ }^{33}$

\subsection{Diagonal Terms}

Here, the diagonal terms of $N$-state-EVB model in eq 1 are described.

\subsubsection{Ionic VB State}

The EVB Hamiltonians for the ionic VB state $(i=1,2, \cdots, N-1$ in Figure 1) are written by

$$
h_{i i}=U^{\mathrm{H}_{3} \mathrm{O}^{+}}+U^{\mathrm{H}_{2} \mathrm{O}}+U^{\mathrm{H}_{3} \mathrm{O}^{+} / \mathrm{H}_{2} \mathrm{O}}+U^{\mathrm{F}^{-} / \mathrm{H}_{3} \mathrm{O}^{+}}+U^{\mathrm{F}^{-} / \mathrm{H}_{2} \mathrm{O}}
$$

where the intermolecular interaction between $\mathrm{F}^{-}$and $\mathrm{H}_{3} \mathrm{O}^{+}$is represented by the following form

$$
U^{\mathrm{F}^{-} / \mathrm{H}_{3} \mathrm{O}^{+}}=U_{\mathrm{F}-}^{\mathrm{LJ}} / \mathrm{H}_{3} \mathrm{O}^{+}+U_{\mathrm{H}^{+} \mathrm{O}_{\mathrm{w}}}^{\mathrm{LJ}}+U_{\mathrm{F}^{-} / \mathrm{H}_{3} \mathrm{O}^{+}}^{\mathrm{Cou}}+U_{\mathrm{F}^{-} \mathrm{O}}^{\mathrm{rep}}+U_{\mathrm{F}^{-} \mathrm{H}^{+}}^{\mathrm{rep}}
$$

with the repulsive potential between $\mathrm{F}^{-}$and $\mathrm{O}$ of $\mathrm{H}_{3} \mathrm{O}^{+}$

$$
U_{\mathrm{F}^{-} \mathrm{O}}^{\mathrm{rep}}=B \exp \left[-b\left(r_{\mathrm{FO}}-d_{\mathrm{FO}}^{0}\right)\right]
$$

and the repulsive potential between $\mathrm{F}^{-}$and $\mathrm{H}^{+}$of $\mathrm{H}_{3} \mathrm{O}^{+}$

$$
U_{\mathrm{F}^{-} \mathrm{H}^{+}}^{\mathrm{rep}}=C \exp \left[-c\left(r_{\mathrm{FH}}-d^{0}\right)\right] .
$$

The diagonal terms for water and hydronium ion molecules $\left(U^{\mathrm{H}_{3} \mathrm{O}^{+}}+U^{\mathrm{H}_{2} \mathrm{O}}+U^{\mathrm{H}_{3} \mathrm{O}^{+} / \mathrm{H}_{2} \mathrm{O}}\right.$ in eq 2) are from the MS-EVB3 model. ${ }^{39} U_{\mathrm{AB}}^{\mathrm{LJ}}$ and $U_{\mathrm{AB}}^{\mathrm{Cou}}$ denote the Lennard-Jones (LJ) and Coulomb interactions between $\mathrm{A}$ and $\mathrm{B}$ atoms. We use the $\mathrm{LJ}$ for $\mathrm{F}^{-}$of Hummer et al. ${ }^{40}$ and added the $\mathrm{LJ}$ interactions between $\mathrm{F}^{-}$and other oxygen atoms by the Lorentz-Berthelot combining rules $\epsilon_{i j}=\sqrt{\epsilon_{i i} \epsilon_{j j}}$ and $\sigma_{i j}=\left(\sigma_{i i}+\sigma_{j j}\right) / 2$. Likewise, $U^{\mathrm{F}^{-} / \mathrm{H}_{2} \mathrm{O}}$ is evaluated as the sum of the $\mathrm{LJ}$ and Coulomb interactions. 


\subsubsection{Covalent VB State}

The diagonal matrix element for the covalent HF VB state and its interaction with the water molecules ( $N$ in Figure 1$)$ is described as

$$
h_{N N}=U^{\mathrm{HF}}+U^{\mathrm{H}_{2} \mathrm{O}}+U^{\mathrm{HF} / \mathrm{H}_{2} \mathrm{O}} .
$$

$U^{\mathrm{HF}}$ is the HF stretching potential modeled by the Morse potential of the form

$$
U^{\mathrm{HF}}=D_{\mathrm{HF}}\left\{1-\exp \left[-\alpha_{\mathrm{HF}}\left(r_{\mathrm{HF}}-r_{\mathrm{HF}}^{\mathrm{eq}}\right)\right]\right\}^{2},
$$

in which the parameters were optimized as $\alpha_{\mathrm{HF}}=1.34675 a_{0}^{-1}, D_{\mathrm{HF}}=0.178645$ hartree, $r_{\mathrm{HF}}^{0}=1.81599 a_{0}$ ( $a_{0}$ is the bohr radius). In eq 6 the intermolecular potential between $\mathrm{HF}$ and $\mathrm{H}_{2} \mathrm{O}$ is given by

$$
U^{\mathrm{HF} / \mathrm{H}_{2} \mathrm{O}}=U_{\mathrm{FO}}^{\mathrm{LJ}}+U_{\mathrm{H}^{\mathrm{F}} \mathrm{O}_{\mathrm{w}}}^{\mathrm{LJ}}+U_{\mathrm{HF} / \mathrm{H}_{2} \mathrm{O}}^{\mathrm{Cou}}+U_{\mathrm{FO}_{\mathrm{w}}}^{\mathrm{rep}}+U_{\mathrm{H}^{\mathrm{F}} \mathrm{O}_{\mathrm{w}}}^{\mathrm{rep}} .
$$

Here, we have used the same charges and LJ parameters for the intermolecular potential of hydrogen fluoride as used by Laage et al. ${ }^{41} \mathrm{H}^{\mathrm{F}}$ means the $\mathrm{H}$ atom bonded to $\mathrm{F}$. $U_{\mathrm{FO}}^{\mathrm{rep}}$ and $U_{\mathrm{HO}}^{\mathrm{rep}}$, respectively, denote the repulsive potential between $\mathrm{F}$ and $\mathrm{O}$ atoms

$$
U_{\mathrm{FO}_{\mathrm{w}}}^{\mathrm{rep}}=B_{\mathrm{F}} \exp \left[-b_{\mathrm{F}}\left(r_{\mathrm{FO}_{\mathrm{w}}}-d_{\mathrm{FO}}^{0}\right)\right]
$$

and $\mathrm{O}$ and $\mathrm{H}^{*}(\mathrm{H}$ atom in $\mathrm{HF}$, see Figure 5) atoms

$$
U_{\mathrm{H}^{\mathrm{F}} \mathrm{O}_{\mathrm{w}}}^{\mathrm{rep}}=C_{\mathrm{F}} \exp \left[-c_{\mathrm{F}}\left(r_{\mathrm{H}^{\mathrm{F}} \mathrm{O}_{\mathrm{w}}}-d^{0}\right)\right] .
$$

The constant term $V_{\text {const }}^{\text {rep }}$ compensates for an excess stabilization of the hydronium- $\mathrm{F}^{-}$states caused by Coulombic interactions between the donor fluorine atom and acceptor hydrogen atoms compared with the ab initio results for small $\mathrm{HF}-\mathrm{H}_{2} \mathrm{O}$ complex (see section 2.4).

\subsection{Off-Diagonal Terms}

The off-diagonal terms are expressed as

$$
h_{i j}=V_{\mathrm{const}}^{i j} A\left(R_{\mathrm{FO}}, q\right),
$$

where $q$ is the proton transfer coordinate defines as ${ }^{31,32}$

$$
q=r_{\mathrm{FH}, \mathrm{FO}}-\frac{r_{\mathrm{FO}}}{2}\left(r_{\mathrm{sc}}^{0}-\frac{r_{\mathrm{FO}}-r_{\mathrm{FO}}^{0}}{15}\right)
$$

in which $r_{\mathrm{FH}, \mathrm{FO}}=\mathbf{r}_{\mathrm{FH}} \cdot \tilde{\mathbf{r}}_{\mathrm{FO}}$ is the distance between the fluorine and the shared hydrogen projected along the $\mathrm{FO}$ vector and $r_{\mathrm{sc}}^{0}$ and $r_{\mathrm{FO}}^{0}$ are empirical parameters. $A\left(R_{\mathrm{FO}}, q\right)$ in eq 11 is given by

$$
\begin{aligned}
A\left(R_{\mathrm{FO}}, q\right)= & \exp \left(-\gamma q^{2}\right)\left\{1+P \exp \left[-k\left(R_{\mathrm{FO}}-D_{\mathrm{FO}}\right)^{2}\right]\right\} \\
& \left\{\frac{1-\tanh \left[\beta\left(R_{\mathrm{FO}}-R_{\mathrm{FO}}^{0}\right)\right]}{2}+P^{\prime} \exp \left[-\alpha\left(R_{\mathrm{FO}}-r_{\mathrm{FO}}^{0}\right)\right]\right\}
\end{aligned}
$$

where $\gamma, P, k, D_{\mathrm{FO}}, \beta, R_{\mathrm{FO}}^{0}, P^{\prime}, \alpha$, and $r_{\mathrm{FO}}^{0}$ are empirical parameters. ${ }^{39}$ 
Table 1: EVB Force Field Parameters for HF and Potential Parameters for the Flexible Hydrogen Fluoride.

\begin{tabular}{lrllrl}
\hline$C$ & 4.57157 & $\mathrm{kcal} / \mathrm{mol}$ & $c$ & 2.1 & $\AA^{-1}$ \\
$C_{\mathrm{F}}$ & 36.5726 & $\mathrm{kcal} / \mathrm{mol}$ & $c_{\mathrm{F}}$ & 2.1 & $\AA^{-1}$ \\
$d^{0}$ & 1.2 & $\AA$ & $V_{\text {const }}$ & -30.1666 & $\mathrm{kcal} / \mathrm{mol}$ \\
$\gamma$ & 1.83029 & $\AA^{-2}$ & $P$ & 0.116363 & \\
$k$ & 9.56215 & $\AA^{-2}$ & $D_{\mathrm{FO}}$ & 2.94 & $\AA^{-1}$ \\
$B$ & 11.26 & $\mathrm{kcal} / \mathrm{mol}$ & $b$ & 1.1 & $\AA^{-1}$ \\
$B_{\mathrm{F}}$ & 0.563 & $\mathrm{kcal} / \mathrm{mol}$ & $b_{\mathrm{F}}$ & 0.55 & $\AA^{-1}$ \\
$\beta$ & 6.01792 & $\AA^{-1}$ & $R_{\mathrm{FO}}^{0}$ & 3.1 & $\AA^{-1}$ \\
$P^{\prime}$ & 10.8831 & $\AA^{-1}$ & $d_{\mathrm{FO}}^{0}$ & 2.5 & $\AA^{-}$ \\
$\alpha$ & 10.0381 & $\AA^{-1}$ & $V_{\text {const }}^{\text {rep }}$ & -220.753 & $\mathrm{kcal} / \mathrm{mol}$ \\
$r_{\mathrm{FO}}^{0}$ & 1.81364 & $\AA^{-1}$ & & & \\
\hline
\end{tabular}

\subsection{Quantum Effective Potential of Mean Force}

The NQE plays an important role in both the vibrational relaxation and the PT. Therefore, we fit the ab initio potential $U_{\mathrm{AI}}(R, r)$ into the quantum effective potential of mean force represented as $^{22-24}$

$U_{\text {eff }}(R, r ; m, T)=-k_{\mathrm{B}} T \ln \int d \mathbf{x} \delta\left(r-x_{c}\right) \exp \left\{-\frac{1}{k_{\mathrm{B}} T}\left[\sum_{i=1}^{P} \frac{m}{2} \omega_{P}^{2}\left(x_{i+1}-x_{i}\right)^{2}+\frac{U_{\mathrm{AI}}\left(R, x_{i}\right)}{P}\right]\right\}$

where $P$ is the number of beads, $\omega_{P}=k_{\mathrm{B}} T \sqrt{P} / \hbar, x_{1}, \cdots, x_{P}$ are the discretized thermal-path coordinates, and $x_{c}=\sum_{i=1}^{P} x_{i} / P$ is the path centroid $\left(x_{P+1}=x_{1}\right)$.

The PES of $\mathrm{HF} / \mathrm{H}_{2} \mathrm{O}$ dimer is shown in Figure 2a, calculated from CCSD(T)/aug-cc-pVTZ using the optimized geometries from MP2/aug-cc-pVTZ with the program GAMESS. ${ }^{42}$ The PESs were calculated by scanning the position of the central hydrogen at the interval of 0.05 $\AA$ between the proton accepting water oxygen and HF fluorine atoms, the latter being held at separations $\left(d_{\mathrm{FO}}\right)$ varying from $2.2 \AA$ to $2.8 \AA$ at the interval of $0.2 \AA$, while all other atoms were allowed to relax.

$U_{\text {eff }}(R, r ; m, T)$ has been computed via Metropolis Monte Carlo integration by umbrella sampling ( $m=1.008 \mathrm{~g} / \mathrm{mol}, T=300 \mathrm{~K}$, and $P=30$ beads) on the same grid points as $U_{\mathrm{AI}}(R, r)$, which is displayed in Figure $2 \mathrm{~b}$. Finally, the lowest eigenvalue for the Hamiltonian matrix was fit to reproduce the effective path integral PES, while some parameters that we could guess reliably were manually adjusted. The result is shown in Figure 2c, which shows a reasonable agreement, the parameters of which are summarized in Table 1. The PES for $R_{\mathrm{FO}}=2.6 \AA$, which is the most relevant in the bulk simulation as seen in section 3.2 , is fitted well.

\subsection{Equilibrium Simulation Details}

A cubic box of dimensions $L=15.74 \AA$ in each direction and $129 \mathrm{H}_{2} \mathrm{O}+\mathrm{HF}$ were used, the density resulting in $0.997 \mathrm{~g} / \mathrm{cm}^{3}$, corresponding to the molar acid concentration, $0.482 \mathrm{~mol} / \mathrm{L}$. The periodic boundary condition was employed and the long-range Coulombic forces were 

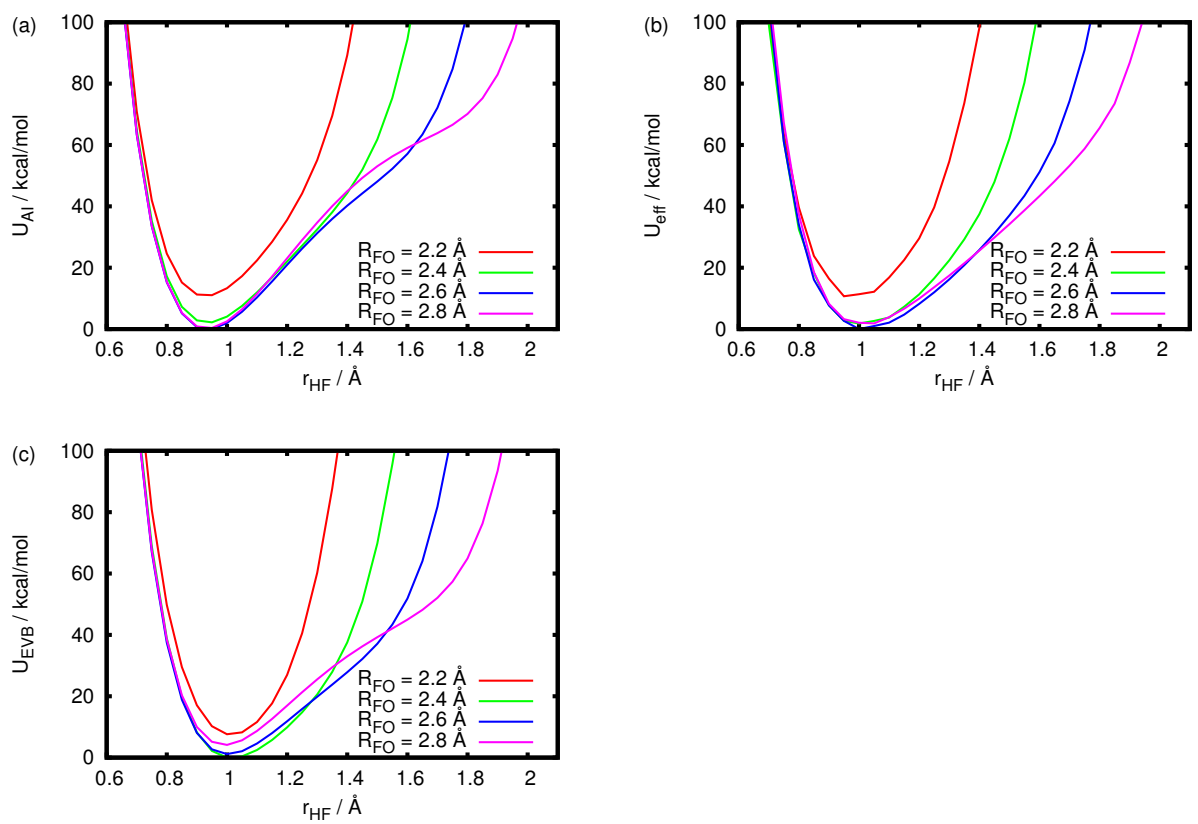

Figure 2: (a) Ab initio PES $U_{\mathrm{AI}}$ for the $\mathrm{HF} / \mathrm{H}_{2} \mathrm{O}$ dimer using $\mathrm{CCSD}(\mathrm{T}) / \mathrm{aug}$-cc-pVTZ with the optimized geometry by MP2/aug-cc-pVTZ. (b) Effective path integral PES $U_{\text {eff }}$ for the same cluster, computed based on eq 14. (c) EVB fit of (b) $U_{\mathrm{EVB}}$, viz., the lowest eigenvalues of eq 1 for the same configurations.

treated by the Ewald summation. The real space screening factor of $\kappa=6 / L$ was used, and the $k$-space was expanded in total of $629 k$-vectors. A cutoff distance of $L / 2$ was applied for the LJ with smoothing and real space electrostatic interactions. The MD time step for the velocity Verlet integrator was $0.1 \mathrm{fs}$. The temperature was set to $300 \mathrm{~K}$ using the Berendsen thermostat for the equilibration run. ${ }^{43}$ By use of the EVB model with the NQE, 100-ps simulations were carried out in the NVE ensemble for the production run.

\section{Results and Discussion}

\subsection{Free Energy of Reaction}

The calculated free energy surface is shown in Figure 3. This was calculated by the free energy perturbation method with an intermediate EVB Hamiltonian $H_{N N}+\lambda\left(H_{11}-H_{N N}\right)$ (where $|1\rangle$ is the VB state with the lowest eigenvalue among ionic VB states) with $\lambda$ varying from 0 to 1 . For each perturbation, trajectories were equilibrated for $100 \mathrm{ps}$ and the data was collected at constant temperature for a period of $100 \mathrm{ps}$.

The calculated reaction free energy is $4.09 \mathrm{kcal} / \mathrm{mol}$. The experimental free energy of the dissociation process can be estimated from the acidity constants of $\mathrm{HF}\left(\mathrm{p} K_{\mathrm{a}}=3.15\right)^{1}$ with $\Delta G=2.3 R T \mathrm{p} K_{\mathrm{a}}(\mathrm{HF})$, resulting in $4.32 \mathrm{kcal} / \mathrm{mol}$. The reasonable agreement with the experiment is the consequence of the inclusion of the NQE by fitting the effective path integral PES in Figure $2 b$.

The calculated activation energy is $\Delta G^{\ddagger}=6.33 \mathrm{kcal} / \mathrm{mol}$. This gives the transition state theory (TST) reaction rate of $k_{\mathrm{TST}}=\left(k_{\mathrm{B}} T / h\right) \exp \left(-\Delta G^{\ddagger} / k_{\mathrm{B}} T\right)=1.53 \times 10^{8} \mathrm{~s}^{-1}$, which is 


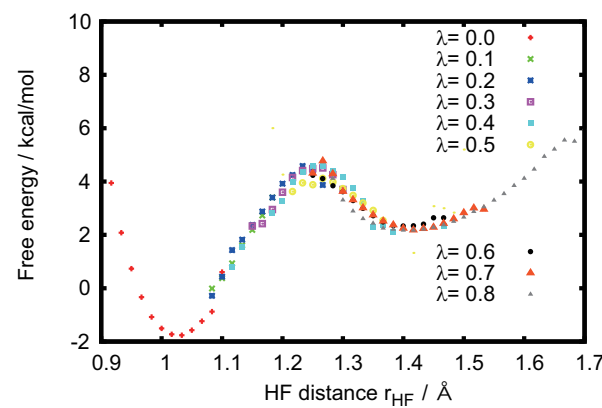

Figure 3: Free-energy surface as a function of the HF distance calculated by the free-energy perturbation method with an intermediate EVB Hamiltonian $H_{N N}+\lambda\left(H_{11}-H_{N N}\right)$.

about twice the experimental value $7 \times 10^{7} \mathrm{~s}^{-1} .^{2}$ The agreement appears reasonable for the thermal rate constant that is sensitive to the exponential factor. Since the experiment includes contributions from the PTs beyond the first solvation shell, ${ }^{44}$ the actual rate for the formation of contact ion pair can be even closer to the calculated value.

To further discuss the reaction rate, the recrossing effect can be estimated from the GroteHynes transmission coefficient $\kappa_{\mathrm{GH}}$ computed by

$$
\kappa_{\mathrm{GH}}=z_{r} / \omega_{\mathrm{b}}, z_{r}^{2}+z_{r} \hat{\zeta}\left(z_{r}\right)-\omega_{\mathrm{b}}^{2}=0
$$

where $\hat{\zeta}(z)$ is the Laplace transform of the friction kernel $\zeta(t)$ of the generalized Langevin equation. ${ }^{45} \omega_{\mathrm{b}}$ is the barrier frequency given by ${ }^{46}$

$$
\omega_{\mathrm{b}}=\Omega\left[\frac{\lambda}{\sqrt{s_{\ddagger}^{2}+4 C^{2}}}\left(1-\frac{s_{\ddagger}^{2}}{s_{\ddagger}^{2}+4 C^{2}}\right)-1\right]^{1 / 2} .
$$

$C, \lambda, s_{\ddagger}$, and $\Omega$ are the proton coupling, the reorganization energy, the energy gap at the barrier top, and the solvent frequency, respectively. The calculation of $\zeta(t)$ and $\Omega$ has been described in ref 47, which yielded $\Omega=540 \mathrm{~cm}^{-1}$. By use of $C=0.82 \mathrm{kcal} / \mathrm{mol}$ and $\lambda=16.8 \mathrm{kcal} / \mathrm{mol}$ from ref 3 and $s_{\ddagger}=0 \mathrm{kcal} / \mathrm{mol}$, we find $\omega_{\mathrm{b}}=1642 \mathrm{~cm}^{-1}$ and $\kappa_{\mathrm{GH}}=0.907$. This $\kappa_{\mathrm{GH}}$ close to unity comes from the rather high value of $\omega_{\mathrm{b}}$ which is off the main region of the friction $\hat{\zeta}(z) \cdot{ }^{47}$ In the same context, the recrossing effect of the HF stretch is also expected to be small. Therefore, it is difficult here to discriminate the solvent motion and the HF stretch for the major reaction coordinate.

We can also evaluate the PT rate by ${ }^{48}$

$$
k=\frac{2 P_{\mathrm{LZ}}}{1+P_{\mathrm{LZ}}} \frac{\Omega}{2 \pi} \exp \left(-\Delta G^{\ddagger} / k_{\mathrm{B}} T\right) .
$$

Here, $P_{\mathrm{LZ}}$ is the Landau-Zener probability given by $P_{\mathrm{LZ}}=1-\exp \left(-2 \pi C^{2} / \hbar \Omega \sqrt{2 k_{\mathrm{B}} T \lambda}\right)$. This model thus describes the reaction by the surface-crossing induced along the solvent coordinate dynamics. With the same parameters as above, we find $2 P_{\mathrm{LZ}} /\left(1+P_{\mathrm{LZ}}\right)=0.63$ and $k=$ $3.96 \times 10^{7} \mathrm{~s}^{-1}$ which is 0.57 times smaller than the experimental rate.

\subsection{Radial Distribution Function}

To examine the solvent structure associated with the two minima on the free energy surface, the RDFs for $\mathrm{HF}\left(\mathrm{FO}, \mathrm{H}^{\mathrm{F}} \mathrm{F}\right.$ and $\mathrm{FH}, \mathrm{H}^{\mathrm{F}} \mathrm{H}$, and $\mathrm{H}^{\mathrm{F}} \mathrm{O}$, where $\mathrm{H}^{\mathrm{F}}$ means the $\mathrm{H}$ atom bonded or 

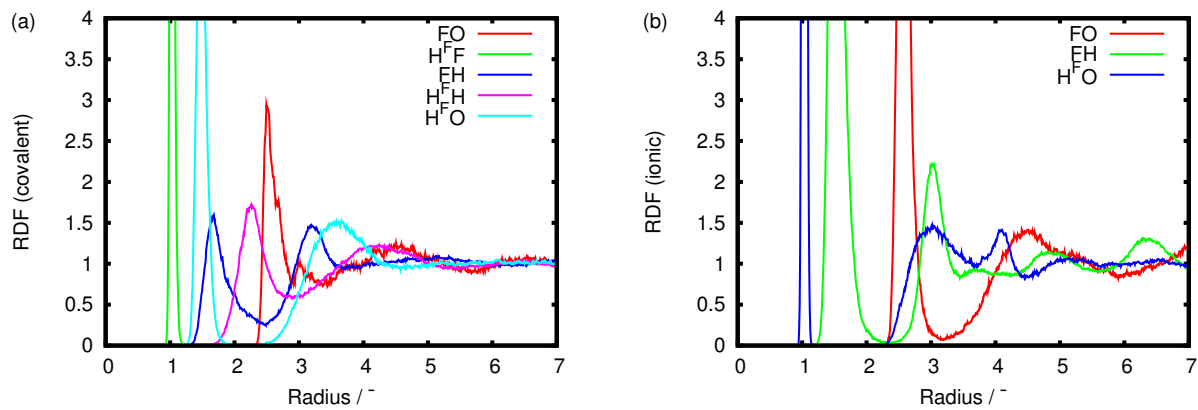

Figure 4: RDFs of $\mathrm{HF}$ in water for the (a) covalent and (b) ionic states (FO, $\mathrm{H}^{\mathrm{F}} \mathrm{F}$ and $\mathrm{FH}, \mathrm{H}^{\mathrm{F}} \mathrm{H}$, and $\mathrm{H}^{\mathrm{F}} \mathrm{O}$, where $\mathrm{H}^{\mathrm{F}}$ means the $\mathrm{H}$ atom bonded or nearest-neighbor to $\mathrm{F}$ atom).

nearest-neighbor to $\mathrm{F}$ atom) are calculated and shown in Figure 4 for the (a) covalent and (b) ionic states. We show the configuration and labels of the HF and water molecules in Figure 5. The water molecule with the asterisk superscript (hereafter, referred to $w^{*}$ ) is that directly H-bonded to the HF molecule. The RDFs for the covalent state are obtained from the equilibrium MD simulation using the ground-state Hellman-Feynman forces since PT rarely occurs at equilibrium. The RDFs for the ionic state are obtained by the simulation with $\lambda=0.8$ in the free energy perturbation method. The choice of $\lambda=0.8$ is based on the free energy curve in Figure 3, which correctly samples the product (contact ion pair) state.

For the covalent state, the HF distance $1.03 \AA$ in our calculation, $\mathrm{H}^{\mathrm{F} F}$ in Figure $4 \mathrm{a}$, is quite close to that calculated by the CP MD simulation $1.06 \AA^{44,49}$ but longer than that by the reference interaction site model self-consistent field (RISM-SCF) simulation $0.94 \AA .{ }^{50}$ The HF molecule forms a strong H-bond to $\mathrm{w}^{*}$ so that the first F-O RDF is split into two: $2.50 \AA$ and $3.00 \AA$ peaks corresponding to $\mathrm{FH} \cdots \mathrm{O}^{*} \mathrm{H}_{2}^{*}$ and $\mathrm{HOH} \cdots \mathrm{FH}$. However, the latter peak is smaller than the CPMD simulation and therefore seems to be merged into the former peak in part. The coordination number of fluorine (with the FO distance less than $3.2 \AA$ ) is 3.33 compared to 3.2 by the CPMD simulation. In contrast, the RISM-SCF simulation observed the single first peak and the longer FO distance $\left(R_{\mathrm{FO}} \sim 3 \AA\right.$ ). The first peak in FH RDF corresponds to the $\mathrm{H}$ atom that is H-bonded to the F atom and is consistent with the CPMD and RISM-SCF calculations. The first peaks in $\mathrm{H}^{\mathrm{F}} \mathrm{H}(2.26 \AA)$ and $\mathrm{H}^{\mathrm{F}} \mathrm{O}(1.48 \AA)$ RDFs shift inward compared to the RISMSCF calculation $(2.5 \AA$ and $1.8 \AA$, respectively). This arises from the stronger H-bond in our calculation than the RISM-SCF calculation. On the other hand, the position of the second peak agrees well with that from the RISM-SCF calculation.

For the ionic state, the first peak of FO RDF $2.57 \AA$ in our calculation is slightly longer than the CPMD simulation $2.49 \AA$ but sharper and shorter than that by the RISM-SCF simulation 2.8 $\AA$. By consideration that both the first peak of FH RDF at $1.51 \AA$ and the first peak of $\mathrm{H}^{\mathrm{F}} \mathrm{O}$ RDF at $1.03 \AA$ shift outward compared with the CPMD results by approximately $0.1 \AA$, the distance of the contact ion pair $\mathrm{F}^{-}$and $\mathrm{H}_{3} \mathrm{O}^{+}$is slightly longer in our model.

\subsection{Tetrahedral and Translational Order Parameters}

To obtain a microscopic picture on the H-bond topology we calculate the local tetrahedral order parameter $^{25,26}$

$$
Q_{k}=1-\frac{3}{8} \sum_{i}^{3} \sum_{j=i+1}^{4}\left[\cos \psi_{i k j}+\frac{1}{3}\right]^{2},
$$




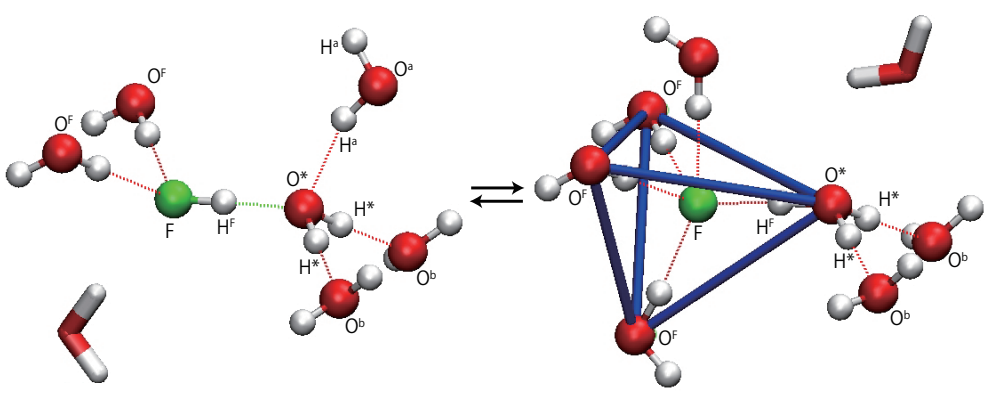

Figure 5: Schematic illustration with labels of $\mathrm{HF}$ and water molecules in the vicinity of the proton accepting water and fluorine atom in the course of PT. The blue bonds between oxygen atoms are attached to display the formation of the local tetrahedral order. The dotted lines represent H-bonds. The molecules that are not so close to be $\mathrm{H}$-bonded but are included in the calculation of the tetrahedral order parameter in eq 18 are drawn by the stick model. These graphics were created using VMD. ${ }^{33}$

and the translational order parameter

$$
t_{\mathrm{X}}^{*}=\int_{0}^{L / 2}\left|g_{\mathrm{XO}}(r)-1\right| d r,
$$

to quantify how the water molecules are arranged and reorganized. Here, $\psi_{i k j}$ is the angle formed by the molecule $k$ and its nearest-neighbors $i$ and $j$, and $g_{\mathrm{XO}}(r)$ is the oxygen/fluorineoxygen $\operatorname{RDF}\left(\mathrm{X}=\mathrm{O}^{(*)} / \mathrm{F}\right)$. In the calculation of Equation 18, the four nearest neighbor oxygen or fluorine atoms are selected on the basis of the distances. For example, if the number of the water molecules in the first solvation shell, as in the left panel of Figure 5, one water molecule in the second solvation shell, is also included in the summation of eq 18. In this case, the calculated $Q$ is small because of the low tetrahedrality. If, on the other hand, the number of the water molecules in the first solvation shell is more than four, only the nearest four water molecules are included in the summation of eq 18. Q's are often used to quantify structural order for the anomalous structural properties of pure water over a range of temperature and pressure values and has been shown to pick up differences in water structure induced by an antifreeze protein. ${ }^{51}$ Therefore, these two measures are expected to offer information on the anomalous structural properties of the HF acid dissociation reaction.

The results of the tetrahedral (with variances) and translational order parameters are summarized in parts a and $b$ of Figure 6, respectively. In this calculation, we sampled the order parameters around $\mathrm{HF}$ and $\mathrm{w}^{*}$ as in section 3.2 for the covalent and ionic states. The transition state means the umbrella sampling simulation with $\lambda=0.5$ in the free-energy perturbation method. $Q_{\mathrm{O}^{*}}$ and $Q_{\mathrm{O}^{\mathrm{F}}}$ increases at the transition state and, in the product state, return to much the same value as in the reactant state. On the other hand, $Q_{\mathrm{F}}$ increases significantly from reactant to product states. Moreover, only $Q_{\mathrm{O}^{\mathrm{a}+\mathrm{b}}}\left(\left[Q_{\mathrm{O}^{\mathrm{a}}}+Q_{\mathrm{O}^{\mathrm{b}}}\right] / 2\right)$ gradually decreases. By comparison with $Q \sim 0.63$ and $t^{*} \sim 0.39$ for pure water at ambient condition, ${ }^{25,26,52}$ the present results indicate that, upon PT, $\mathrm{H}$-bonds around the fluorine atom are formed to increase its local tetrahedrality, This is one of the factors decreasing the entropy in the product state. This can also be seen in the small variances of $Q_{\mathrm{F}}$ at the transition and product states.

In the reactant state, the water molecule in the second solvation shell (the water molecules drawn by the stick model in Figure 5) is included to calculate Equation 18 and deform a tetrahedral $\mathrm{H}$-bond network. However, since the coordination number of the $\mathrm{F}$ atom increase from 

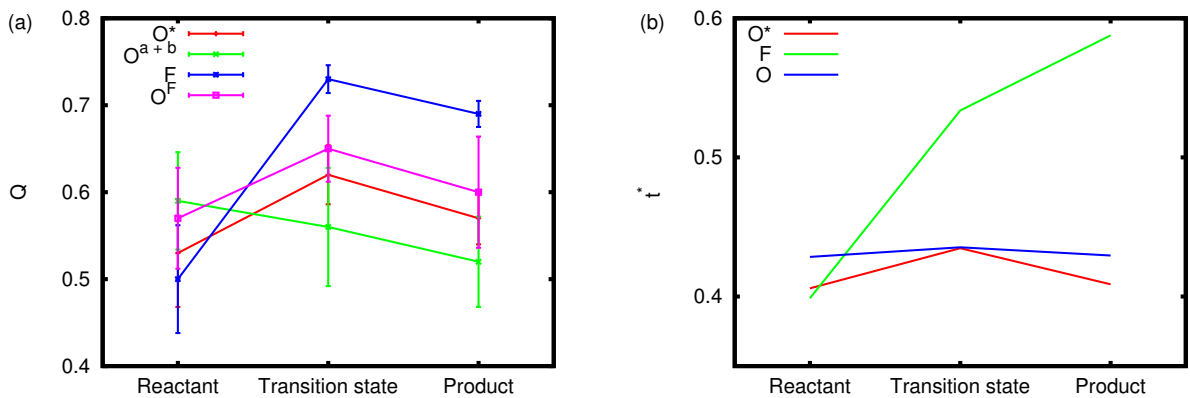

Figure 6: (a) Tetrahedral order parameters defined in eq 18 at reactant, transition, and product states around $\mathrm{O}^{*}, \mathrm{O}^{\mathrm{a}+\mathrm{b}}, \mathrm{F}$, and $\mathrm{O}^{\mathrm{F}}$ atoms in Figure 5. The error bars of the tetrahedral order parameters are the variances. (b) Translational order parameters with $\mathrm{X}=\mathrm{O}^{*}, \mathrm{~F}$, and $\mathrm{O}$ in eq 19. For the labels of atoms and the definitions of the reactant, transition, and product states, see Figure 5 and text.

3.33 to 5.21 during the PT with FO distance less than $3.2 \AA$, about two water molecules become available to from the tetrahedral $\mathrm{H}$-bond network, thus increasing $Q_{\mathrm{F}}$ as shown in Figure 5. The decrease of $Q_{\mathrm{O}^{\mathrm{a}+\mathrm{b}}}$ is a consequence of the formation of a hydronium ion, which lead to destruction of the tetrahedral H-bond network further than the first solvation shell. On the other hand, the small change of $Q_{\mathrm{O}^{*}}$ between the reactant and product states comes from the inclusion of the water molecule drawn by the stick model in the right panel of Figure 5. This molecule is not $\mathrm{H}$-bonded to $\mathrm{w}^{*}$ and yet remains near $\mathrm{w}^{*}$ with the distance to $\mathrm{O}^{*}$ ranging from 2.75 to $3.5 \AA .{ }^{39}$

Moreover, Figure $6 \mathrm{~b}$ shows that $t_{\mathrm{F}}^{*}$ gradually increases, $t_{\mathrm{O}^{*}}^{*}$ slightly increases, and $t_{\mathrm{O}}^{*}$ remains almost constant. This indicates that the order of $\mathrm{H}$-bond structure along the radial direction increases significantly around the $\mathrm{F}$ atom and slightly around the $\mathrm{O}^{*}$ atom. The increase can be explained by the RDF of FO in Figure 4, where that in the ionic state has deeper peaks and troughs. In other words, upon PT $\mathrm{F}^{-}$anion so strongly attracts the first solvation shell that a clear separation between the first and second solvation shell appears. It is noted that $Q \mathrm{~s}$ have some peaks at the transition state, and yet $t^{*} \mathrm{~s}$ do not. This indicates that to initiate the reaction and overcome the activation barrier large angular H-bond rearrangements are more essential than translational ones.

We now discuss the change in the H-bond topology associated with the weak acidity in a dilute hydrofluoric acid solution at ambient temperature. The $\mathrm{F}$ atom is the smallest one among halogens and in the same period as $\mathrm{O}$ atom, which would have the best radius among halogen anions to form a (tetrahedral) $\mathrm{H}$-bond network in water as suggested in the RISMSCF simulation. ${ }^{50}$ On the other hand, the other structure-breaking halide anions $\left(\mathrm{Cl}^{-}, \mathrm{Br}^{-}\right.$, $\mathrm{I}^{-}$) are expected to show a lower tetrahedrality around themselves. Our results present the possibility that the local tetrahedral and translational order parameter could be a measure of the H-bond topology changes associated with the entropy change in the PT, possibly for a series of hydrohalic acids. We are currently working on comparison with other hydrohalic acids and quantitative calculations of the entropy change, ${ }^{53,54}$ which will be reported in due course.

\section{Conclusion}

In the present work, we have performed the MS-EVB MD simulations of a dilute hydrofluoric acid solution at ambient temperature to study the hydration structure during the PT associated 
with its weak acidity.

The simulated activation energy and reaction free energy by the developed MS-EVB model were found to agree reasonably with experiment. In addition, the simulated RDFs showed similar results as other simulations, such as the CPMD and RISM-SCF simulations.

The local tetrahedral and translational order parameters were calculated to obtain a microscopic picture on the H-bond topology. The parameters around the fluorine atom were found to significantly increase in the transition and product states of HF dissociation reaction compared to the other atoms. This suggests that for the proton transfer to occur the change in H-bond topology is necessary especially near the $\mathrm{F}$ atom. Therefore, the $\mathrm{F}^{-}$anion actually "makes" the hydration structure in water in the PT reaction. On the other hand, the local tetrahedral order parameters around the water molecules in the first solvation shell of the proton-accepting water decreased, indicating that the $\mathrm{H}_{3} \mathrm{O}^{+}$cation "breaks" the hydration structure in water. In addition, at the transition state of the proton transfer, the tetrahedral order parameters are very large whereas the translational order parameters are not. This suggests that for the proton transfer to occur the large angular rearrangements of the hydrogen-bond topology are more necessary than the translational ones.

\section{Acknowledgment}

T.J. was supported by the Research Fellowship of the Japan Society for the Promotion of Science and the Grants in Aid for Scientific Research (KAKENHI) from the Ministry of Education. K.A. acknowledges supportvj from KAKENHI Nos. 20108017 (" $\pi$-space") and 22550012.

\section{References}

[1] Eigen, M.; Kustin, K. J. Am. Chem. Soc. 1960, 82, 5952-5953.

[2] Eigen, M.; Kruse, W.; Maass, G.; De Maeyer, L. Prog. React. Kinet. 1964, 2, 285-318.

[3] Ando, K.; Hynes, J. T. J. Phys. Chem. B 1997, 101, 10464-10478.

[4] Ando, K.; Hynes, J. T. J. Phys. Chem. A 1999, 103, 10398.

[5] Laasonen, K.; Larrucea, J.; Sillanpää, A. J. J. Phys. Chem. B 2006, 110, 12699-12706.

[6] Paul, M. A.; Lang, F. A. Chem. Rev. 1957, 57, 1-45.

[7] Pauling, L. J. Chem. Educ. 1956, 33, 16-17.

[8] Pauling, L. J. Chem. Educ. 1976, 53, 762-767.

[9] Myers, R. T. J. Chem. Educ. 1976, 53, 17.

[10] Ayotte, P.; Hébert, M.; Marchand, P. J. Chem. Phys. 2005, 123, 184501-184508.

[11] Ho, M.-H.; Klein, M. L.; Kuo, I. F. W. J. Phys. Chem. A 2009, 113, 2070-2074.

[12] Ellis, A. J. J. Chem. Soc. 1963, 4300-4304.

[13] McCoubrey, J. C. Trans. Faraday. Soc. 1955, 51, 743. 
[14] Marcus, Y. Ion Solvation; Wiley: New York, 1958.

[15] Omta, A. W.; Cropman, M. F.; Woutersen, S.; Bakker, H. J. Science 2003, 301, 347.

[16] Bakker, H. J. Chem. Rev. 2008, 108, 1456-1473.

[17] Schmidt, D. A.; Birer, O.; Funkner, S.; Born, B. P.; Gnanasekaran, R.; Schwaab, G. W.; Leitner, D. M.; Havenith, M. J. Am. Chem. Soc. 2009, 131, 18512-18517.

[18] Laage, D.; Hynes, J. T. Proc. Nat. Am. Soc. 2007, 104, 11167-11172.

[19] Warshel, A.; Weiss, R. M. J. Am. Chem. Soc. 1980, 102, 6218-6226.

[20] Villa, J.; Warshel, A. J. Phys. Chem. B 2001, 105, 7887-7907.

[21] Cao, J. S.; Voth, G. A. J. Chem. Phys. 1994, 101, 6168-6183.

[22] Brancato, G.; Tuckerman, M. E. J. Chem. Phys. 2005, 122, 224507-224517.

[23] Wick, C. D.; Dang, L. X. J. Phys. Chem. A 2009, 113, 6356-6364.

[24] Vuilleumier, R.; Borgis, D. J. Phys. Chem. B 1998, 102, 4261-4264.

[25] Errington, J. R.; Debenedetti, P. G. Nature 2001, 409, 318-321.

[26] Kumar, P.; Buldyrev, S. V.; Stanley, H. E. Proc. Nat. Am. Soc. 2009, 106, 22130-22134.

[27] Vuilleumier, R.; Borgis, D. J. Mol. Struct. 1998, 555, 436-437.

[28] Vuilleumier, R.; Borgis, D. J. Phys. Chem. B 1998, 102, 4261.

[29] Vuilleumier, R.; Borgis, D. Chem. Phys. Lett. 1998, 284, 71.

[30] Swanson, J. M. J.; Maupin, C. M.; Chen, H.; Petersen, M. K.; Xu, J.; Wu, Y.; Voth, G. A. J. Phys. Chem. B 2007, 111, 4300-4314.

[31] Cuma, M.; Schmidt, U. W.; Voth, G. A. Chem. Phys. 2000, 258, 187-199.

[32] Cuma, M.; Schmidt, U. W.; Voth, G. A. J. Phys. Chem. A 2001, 105, 2814-2823.

[33] Humphrey, W.; Dalke, A.; Schulten, K. J. Mol. Graphics. 1996, 14, 33.

[34] Giguère, P. A.; Turrell, S. J. Am. Chem. Soc. 1980, 102, 5473-5477.

[35] Giguère, P. A.; Martel, C.; Turrell, S. Chem. Phys. Lett. 1978, 56, 231-234.

[36] Simon, C.; Klein, M. L. Chem. Phys. Chem. 2005, 6, 148-153.

[37] Ayotte, P.; Plessis, S.; Marchand, P. Phys. Chem. Chem. Phys. 2008, 10, 4785-4792.

[38] Thomas, V.; Iftimie, R. J. Phys. Chem. B 2009, 113, 4152-4160.

[39] Wu, Y.; Chen, H.; Wang, F.; Paesani, F.; Voth, G. A. J. Phys. Chem. B 2008, 112, 467-482.

[40] Hummer, G.; Pratt, R.; Garcia, A. F. J. Phys. Chem. 1996, 100, 1206-1215. 
[41] Laage, D.; Demirdjian, H.; Hynes, J. T. Chem. Phys. Lett. 2005, 405, 453-458.

[42] Schmidt, M. W.; Baldridge, K. K.; Boatz, J. A.; Elbert, S. T.; Gordon, M. S.; Jensen, J. H.; Koseki, S.; Matsunaga, N.; Nguyen, K. A.; Su, S.; Windus, T. L.; Dupuis, M.; Montgomery, J. A. J. Comput. Chem. 1993, 14, 1347-1363.

[43] Berendsen, H. J.; Postma, J. P. M.; Vangunsteren, W. F.; Haak, J. R. J. Chem. Phys. 1984, $81,3634-3690$.

[44] Sillanpää, A. J.; Simon, C.; Klein, M. L.; Laasonen, K. J. Phys. Chem. B 2002, 106, 11315-11322.

[45] Grote, R. F.; Hynes, J. T. J. Chem. Phys. 1980, 73, 2715-2732.

[46] Ando, K. J. Chem. Phys. 1994, 101, 2850-2862.

[47] Joutsuka, T.; Ando, K. J. Phys. Chem. A 2010, DOI: 10.1021/jp108413e.

[48] Straub, J. E.; Berne, B. J. J. Chem. Phys. 1987, 87, 6111-6116.

[49] Laasonen, K.; Klein, M. L. Mol. Phys. 1996, 88, 135-142.

[50] Sato, H.; Hirata, F. J. Am. Chem. Soc. 1999, 121, 3460-3467.

[51] Smolin, N.; Daggett, V. J. Phys. Chem. B 2008, 112, 6193-6202.

[52] Jabes, B. S.; Agarwal, M.; Chakravarty, C. J. Chem. Phys. 2010, 132, 234507.

[53] Giuffré, E.; Prestipino, S.; Saija, F.; Saitta, A. M.; Giaquinta, P. V. J. Chem. Theory Comput. 2010, 6, 625-636.

[54] Wang, L.; Abel, R.; Friesner, R. A.; Berne, B. J. J. Chem. Theory Comput. 2009, 5, 14621473. 


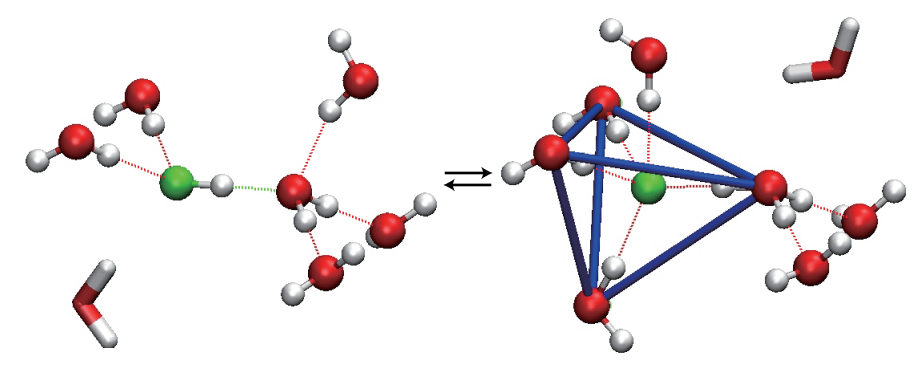

Table of contents graphics 